\title{
PAULO COELHO: ALGUMAS REFLEXÕES SOBRE A SUA RECEPÇÃO NA COSTA DO MARFIM
}

\section{PAULO COELHO: SOME THOUGHTS ON HIS RECEPTION IN COTE D'IVOIRE}

\author{
Brou Angoran Anasthasie* Yéo N'gana**

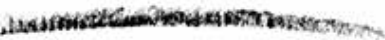 \\ RESUMO
}

Conhecer a si próprio e o desenvolvimento pessoal têm se tornado temáticas, cada vez mais, presentes entre os marfinenses que, por sua vez, vêm dando ao escritor brasileiro Paulo Coelho um lugar de destaque. Nesta investigação, tentamos conduzir um trabalho de campo junto a quatro livrarias, a saber:a Librairie de France-Plateau,a ex-Leader Price,a FNAC-a Cap Nordea Carrefour Siloe, cujo intuito é inquirir o que, deste autor, vem sendo consumido nestes espaços. Nos importa é compreender o percurso dessas obras que, cabe lembrar, são escritas em língua portuguesa e nos chegam através de editores franceses. Em resumo, tecemos reflexões sobre o que consideramos ser uma mediação da mediação nos Estudos da Tradução. Concluímos que embora o interesse pelos livros do Paulo continue crescendo, não traz, por enquanto, luz nenhuma sobre o setor da tradução na Costa do Marfim. O que, consequentemente, outorga à sua recepção um caráter de incompletude.

Palavras-chave: Paulo Coelho. Internacionalização. Tradução e recepção.

\section{ABSTRACT}

Self-awareness and personal development have recently become trendy topics among many Ivorians to whom the Brazilian writer Paulo Coelho begins to play an important role. For the writing of this paper, we carried out a fieldwork in four bookstores which included the following: Librairie de France - Plateau, ex-Leader Price, FNAC-Cap Nord and Carrefour Siloe. We sought to identify the books they mostly sold of this author. It was of paramount importance for us to know the journey of those books, stressing here on the fact that there are written in Portuguese, and then came to us through French editors. In a nutshell, we reflected on what we consider to be a mediation of the mediation in Translation Studies. As a conclusion, we believe that though readers' interest towards Paulo Coelho's writing keeps growing, it is not helping cast a new light on the translation sector in Côte d'Ivoire, and giving the author's reception, an incomplete trait.

Keywords: Paulo Coelho. Internationalization. Translation and reception.

*Professora do Departamento de Estudos Ibéricos e Latino-Americano da Université Félix Houphouët Boigny (Abidjan, Costa do Marfim), Assistente pelo Conseil Africain et Malgache pour l'Enseignement Supérieur, CAMES. Orcid : https://orcid. 4.org/0000-0001-9943-608X

**Professor Substituto do Departamento de Estudos Ibéricos e Latino-Americano da Université Félix Houphouët Boigny (Abidjan, Costa do Marfim). Orcid : https://orcid.org/0000-0002-6172-306 


\section{INTRODUCTION}

Quand l'homme a montré son arrogance, Dieu a détruit la tour de Babel et tous se sont mis à parler des langues différentes. Mais dans Son infinie bienveillance, Il a créé également une sorte de gens qui allait reconstruire ces ponts, permettre le dialogue et la diffusion de la pensée humaine. Cet homme (ou cette femme) dont nous nous donnons rarement la peine de connaître le nom quand nous ouvrons un livre étranger : le traducteur.

\section{Paulo Coelho}

Vue sous un angle technique, la littérature est l'exercice d'un plagiat formel de genres déjà solidement constitués dans la vie réelle du langage. 1

\section{Cristóvão Tezza}

Les métaphores qui font du traducteur unmédiateur(c'est-à-dire, celui qui, connaisseur des deux langues et cultures, s'engage à apaiser les éventuelles tensions sémiques existantes entre parties concernées), un interlocuteur (muni de son biculturalisme, le traducteur se voit légitimement attribué le rôle, si ce n'est de palabreur officiel, de partenaire capable de recevoir les projets culturels des uns pour les soumettre aux autres), un pont (il devrait ainsi, dans une immobilité pourtant mobile, déplacer ou permettre que des discours, messages-idées soient passés d'un espace physico-métaphysique à un autre, sans inclinaison de sa part. Il s'agit ici d'un rythme difficilement tenable. La neutralité comme la fidélité sont, dans le cadre de la traduction littéraire, une chimère qui peut s'avérer préjudiciable.) et enfin un traître (soit, un traduttore traditore. Et, s'il se laisse [em]porter par la mélodie tentatrice qu'est la fidélité ou la neutralité; les idiosyncrasies de l'œuvre, les exigences du client, les dispositions politico-culturelles de la langue d'arrivée, sont susceptibles de le conduire vers une neutralité plutôt infidèle), ne sauraient tarir ni cesser de se réinventer.

Dans le cadre précis de cet article, il convient de remarquer que les différents éléments évoqués, appelons-les éléments motivateurs ou elmots, qui influencent l'inclinaison ou la position politique du traducteur, présentent une forme de duplicité. Autrement dit, dans le cas de la Côte d'Ivoire, ni le public, ni le traducteur, ni les revendeurs - s'agissant ici des librairies: ex-Leader Price, FNAC-Cap Nord, Librairie de France et Carrefour Siloe - n'ont aucune influence directe sur le produit fini. La nature et la qualité du bien consommé sont fonction des elmots des éditeurs français. Ceux-ci pourraient les définir selon l'agenda qu'ils ont pour la Côte d'Ivoire ou pour leur public cible dans sa généralité. Les questions que l'on se pose sont les suivantes: Les traductions consommées en Côte d'Ivoire ont-elles été faites particulièrement pour ce pays? S'agit-il de traductions faites pour le grand public francophone en général?

Ceci dit, dans un premier temps, nous présenterons brièvement Paulo Coelho. Ensuite, nous montrerons, dans des tableaux, les ouvrages que chacune des librairies choisies possédaient de cet auteur. Enfin, bien qu'appartenant à des contextes complètement différents, il n'en demeure pas moins que le parcours triangulaire effectuée par l'œuvre de Paulo Coelho, avant de nous parvenir, nous rappelle un autre aspect de la centralité de la France dans la diffusion des œuvres littéraires en Afrique. Pour ce faire, nous tisserons quelques réflexions sur les possibles elmots d'un tel flux.

\section{PAULO COELHO: BIOGRAPHIE}

Paulo Coelho est actuellement l'auteur brésilien le plus lu et ses œuvres sont traduites en plus de 80 langues et publiées dans 150 pays à travers le monde. Né en 1947, Paulo Coelho est devenu membre de l'Académie Brésilienne des Lettres depuis le 12 Juillet 2002, succédant à Roberto Campos titulaire du fauteuil $\mathrm{n}^{\circ} 21$. Il a toujours évolué dans le monde des arts et des lettres 
au Brésil, en tant que professeur, directeur et auteur de pièces de théâtre, journaliste et compositeur. Il a écrit plusieurs chansons pour de célèbres chanteurs brésiliens notamment: Elis Regina, Rita Lee et Raul Seixas (célèbre chanteur et compositeur de rock) avec qui il a entretenu un partenariat musical plus fructueux et plus long incluant plus d'une soixantaine de compositions, dont quelques succès comme: "Eu nasci há dez mil anos "(je suis né il y a dix mille ans), " Gitta ", etc. En 1972, il a créé la revue 2001 .

Paulo Coelho a vécu une période de Hippie et connu une fascination pour le mysticisme qui l'ont amené à fréquenter plusieurs sectes ésotériques. Après quelques tentatives peu fructueuses en tant qu'écrivain, sa carrière littéraire débutera après un pèlerinage à Santiago de Compostela en Espagne en 1986 que suivra la publication des ouvrages: " O diário de um mago - O Peregrino " (1987), " O Alquimista ". Cette ultime œuvre sera le roman brésilien le plus vendu, déclenchant ainsi, sur le plan national et international, le cycle de succès des œuvres de cet auteur. Succès qui a permis à Paulo Coelho de remporter le "Guinness World Record" de l'auteur le plus traduit de son vivant et de celui ayant signé le plus de contrats avec différentes maisons d'édition.

En 2012, le journaliste Fernando Morais écrit la biographie de Paulo Coelho dans le livre $O$ $\operatorname{Mago}^{2}$ (Le Mage) à partir d'entretiens et de manuscrits réunis pendant plus de quarante ans.

Ce succès mondial de l'auteur étant tributaire de la réception qui l'a provoquée (ou qu'elle a provoqué), il convient de s'interroger sur ses implications politiques.

\section{QUADRE THÉORIQUE}

Tandis que l'histoire de la pensée politique met l'accent sur les diverses compréhensions des textes perçus comme de complexes actes du langage; dans les théories de la réception littéraire, le focus est plutôt mis sur la ou les différentes interprétations et évaluations des textes, surtout, quand ils sont de nature littéraire ou artistique (Thompson, 2001). En traductologie, c'est notamment dans le chevauchement, mieux, le syncrétisme de ces deux approches que réside toute la fortune pour l'histoire de la traduction et, par-dessus tout, pour l'économie du savoir. Parler de la réception implique une ouverture tacite (voulue ou forcée) de la culture d'arrivée. Avec une telle aspiration, au-delà de l'expansion des idées et de l'actualisation de la mappemonde de la littérature dite "universelle", c'est la mise en examen du méridien de Greenwich livresque (Casanova, 2004, 2015) qui est engagée. Soit,

Writers in postcolonial nations on the periphery of international literary space therefore have to struggle not only against the predominance of national politics, as writers in the richest spaces do, but also against international political forces. The external forces exerted upon the least endowed literary spaces today assume the forms of linguistic domination and economic domination (notably in the form of foreign control over publishing), which is why proclamations of national independence do not suffice to eliminate outside pressures. To one degree or another, then, literary relations of power are forms of political relations of power. (CASANOVA, 2004, p. 81)

Autrement dit, ce "centre législateur", pour emprunter un terme casanovien, n'a excellé que dans la production de "dominés littéraires". Cette perspicace lecture de Casanova nous en rappelle une autre, celle du philosophe ivoirien Adé Adiaffi. Celui-ci écrit:

Aucune langue ne naît riche ; c'est l'usage qui l'enrichit. [...] Nous avons à livrer la même bataille, à défendre et à illustrer nos langues ; si nous enterrons nos langues, dans le même cercueil, nous enfouissons à jamais nos valeurs culturelles, toutes nos valeurs culturelles, d'autant plus profondément que [...] la langue reste l'unique archive. (ADIAFFI, 1980, p. 107)

A défaut d'en avoir un qui soit tournant, il n'y a nul besoin de dire qu'il est urgent que d'autres langues postulent ou convergent vers ce "centre". Par son statut même d'instrument de pouvoir (Bourdieu apud Casanova, 2015), la langue dominante et/ou traduisante peut, dans cette République de tyrans, créer chez ces dominés ou annexés littéraires une illusion d'optique toujours périlleuse. La langue, porteuse de la littérature, est au départ frêle et exposée à l'ambition de 
l'oubli. Elle a donc un besoin vital, comme l'écrivait Adiaffi, d'être enrichie par l'usage. En s'érigeant avec le temps en dépositaire de la mémoire collective, la dominer, c'est se distinguer des autres. C'est être un et plusieurs autres à la fois. Les dominés littéraires se trouvent alors dans l'impasse. Soit, ils s'évertuent, dans un temps relativement imparti, à dominer la langue qui jouit déjà d'une place au soleil. Soit, ils s'engagent dans la construction d'une autre qui, dans le temps long, pourra bénéficier de pareil "prestige".

Le premier cas, hautement dépendant du détenteur du capital, leur est imposé sans préavis. Ici, l'administrateur du numéraire est aussi le teneur du littéraire. Sans répit, il est donc attiré par tous les aspects de la vie politique, spirituelle et économique de la société. La domination de la langue du dominé ne peut que susciter chez celui-ci une envie irréfrénable de dominer la langue de sa domination, langue de son assujettissement à travers laquelle il nourrit l'espoir d'une éventuelle renaissance.

Dans le second cas, ce qui importe, c'est la "pertinence" du projet. Thompson (1993) a bien raison lorsqu'il dit qu'il s'agit-là d'un terme relatif, pour dire que tout est pertinent pour quelque chose. La pertinence ici implique: Qui fait usage de quelle langue pour produire quelle littérature et pour qui? En d'autres termes, quel type de société traduit-on pour quel autre type de société (ou horizon d'expectation) dans quel langage et pourquoi? En résumé, ce qui semble traverser toutes ces préoccupations, c'est le rapport que l'on a à l'autre. De quelle image de moi l'autre a-t-il droit, vice-versa?

Que l'on s'accorde ou pas, la langue traduisante jouit toujours d'une certaine forme de domination qui se paie toute spéculation relative au débat target oriented approach versus source oriented approach. Il reste indubitable qu'à la lisière de deux pôles, le sujet-traduisant use de son "pouvoir" pour restructurer les géographies littéraires dont il est le régisseur temporaire. Dans sa qualité de moi-hiatus situé entre notre moi et le moi de l'autre, il possède entre les mains la possibilité de (re)localiser le méridien livresque et d'influencer la réception de l'œuvre (re)produite. Il peut se trouver dans l'interstice directe ou se poster à des frontières subsidiaires (ou annexes) comme c'est le cas des librairies.

\section{LA LIBRAIRIE DE FRANCE, EX-LEADER PRICE, FNAC-CAP NORD ET CARREFOUR SILOE}

Le livre est, dans toute communauté, un évènement. Un tel évènement doit son Salut à la géographie d'interprétation que lui offre les "sujets de perception"3. La transition du statut $\mathrm{d}$ "évènement" (périssable) à celui de "fait" (concret et immuable), exige une fixation ou une exposition continue. Dans sa forme solide, l'évènement devenu fait parce que placé dans un contexte culturel et socio-politique est maintenant nommé sur la base d'un certain nombre de valeurs, et inscrit dans la mémoire vivante ou temporairement inerte de la communauté par l'intermédiaire d'une langue (ou langage). Les bibliothèques et les librairies sont pour ces évènements-faits-mémoires des lieux de promotion de passés présents. Le passage de l'évènementau fait rendu possible certes par des écrivains (ou journalistes) est tributaire à la fois du traducteur et des éditeurs qui lui offrent des branches et des racines qui s'étendent sur d'autres horizons.

Voyons donc comment, dans le cas ivoirien, les faits-mémoires de Paulo Coelho sont disséminés dans la société par l'entremise des librairies susmentionnées. Nous présentons ici les titres que nous avons trouvé dans les stands des librairies choisies pour le présent article. Un formulaire de recherche a été attribué à chaque librairie. Et il se présente comme suit:

Figure 1. Formulaire élaboré par les auteurs 


\section{Formulaire de recherche}

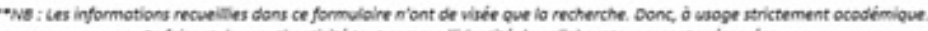
Ce foisant, leur outhentiobi tout comme hidenbie du collaboroteur seront priservies.

Saviez-vous qu'il s'agissait là d'une traduction ?

3. Quelles ceuvres de Paulo Coelho votre librairie vend-elle le plus ?

4. Quels titres de Paulo Coelho avez-vous en Stock et/ou en stand dans votre librairie ? Veuillez Sill vous plait renseigner le tableau ci-après :

\begin{tabular}{|c|c|}
\hline TITRES DISDONIBLES & $\begin{array}{l}\text { QTE EN } \\
\text { STAND }\end{array}$ \\
\hline \multicolumn{2}{|l|}{ Adoultedre } \\
\hline \multicolumn{2}{|l|}{ Alegh } \\
\hline \multicolumn{2}{|l|}{ Amour } \\
\hline \multicolumn{2}{|l|}{ Brida } \\
\hline \multicolumn{2}{|l|}{ Comme le fleuve qui coule } \\
\hline \multicolumn{2}{|l|}{ Mippie } \\
\hline \multirow{2}{*}{\multicolumn{2}{|c|}{$\begin{array}{l}\text { L'alchimiste } \\
\text { La cinguième montarne }\end{array}$}} \\
\hline & \\
\hline \multicolumn{2}{|l|}{ La solitude du vainqueur } \\
\hline \multicolumn{2}{|l|}{ La sarcibre de Portobello } \\
\hline \multicolumn{2}{|l|}{ Le démon et mademoisele Prym } \\
\hline \multicolumn{2}{|l|}{ Le manuserit tetround } \\
\hline \multicolumn{2}{|l|}{ Le pèleqin de Compostelle } \\
\hline \multicolumn{2}{|l|}{ Le zahit } \\
\hline \multirow{2}{*}{\multicolumn{2}{|c|}{$\begin{array}{l}\text { Lespianne } \\
\text { Maktub }\end{array}$}} \\
\hline & \\
\hline \multirow{2}{*}{\multicolumn{2}{|c|}{$\begin{array}{l}\text { Manuel du ruerrier de la lumitre } \\
\text { Onze minutes }\end{array}$}} \\
\hline & \\
\hline Sur le bord de la rivitere Pledra. je me suis assise et jai pleuré & \\
\hline
\end{tabular}

5. Qu'est-ce qui suscite, selon wous, un tel intérêt pour cet écrivain brésilien ? Que vous disent les clients et/cu lecteurs (leur motivation) ?

Nous vous remercions de votre contribution.

Chercheurs requérants.

Au nombre des librairies consultées, ce sont:

FNAC - Cap Nord

\begin{tabular}{|l|r|}
\hline \multicolumn{1}{|c|}{ TITRES DISPONIBLES } & QTE EN STAND \\
\hline Adultère & Indisponible \\
\hline Aleph & Indisponible \\
\hline Amour & Indisponible \\
\hline Brida & 3 \\
\hline Comme le fleuve qui coule & 3 \\
\hline Hippie & 5 \\
\hline L'alchimiste & Indisponible \\
\hline La cinquième montagne & 2 \\
\hline La solitude du vainqueur & Indisponible \\
\hline La sorcière de Portobello & Indisponible \\
\hline Le démon et mademoiselle Prym & 3 \\
\hline Le manuscrit retrouvé & Indisponible \\
\hline Le pèlerin de Compostelle & 1 \\
\hline Le zahir & 4 \\
\hline L'espionne & Indisponible \\
\hline Maktub & Indisponible \\
\hline Manuel du guerrier de la lumière & Indisponible \\
\hline Onze minutes & Indisponible \\
\hline $\begin{array}{l}\text { Sur le bord de la rivière Piedra, je me suis assise et j'ai } \\
\text { pleuré }\end{array}$ & 3 \\
\hline Véronika décide de mourir & 2 \\
\hline
\end{tabular}


$* *$ Lecture du formulairen ${ }^{\circ} 1$ :

Les réponses fournies pour les questions 1 et 2 sont: "Non, pas encore" et "non, je ne savais pas". La réponse 3 fait état de ce que toutes les œuvres de l'auteur suscitent, chez les lecteurs, un grand intérêt. Mais, la libraire qui nous a reçu met un accent particulier sur "L'alchimiste". Pour ce qui concerne l'engouement (question 5), elle évoque comme elmots, le développement personnel et la spiritualité comme tendance actuelle. A noter que la réponse 4 se réfère au tableau $\uparrow$.

Ex-LEADER PRICE - Riviera 4

\begin{tabular}{|l|r|}
\hline \multicolumn{1}{|c|}{ TITRES DISPONIBLES } & QTE EN STAND \\
\hline Adultère & Indisponible \\
\hline Aleph & Indisponible \\
\hline Amour & Indisponible \\
\hline Brida & Indisponible \\
\hline Comme le fleuve qui coule & Indisponible \\
\hline Hippie & Indisponible \\
\hline L'alchimiste & Indisponible \\
\hline La cinquième montagne & Indisponible \\
\hline La solitude du vainqueur & Indisponible \\
\hline La sorcière de Portobello & Indisponible \\
\hline Le démon et mademoiselle Prym & Indisponible \\
\hline Le manuscrit retrouvé & Indisponible \\
\hline Le pèlerin de Compostelle & \\
\hline Le zahir & Indisponible \\
\hline L'espionne & Indisponible \\
\hline Maktub & Indisponible \\
\hline Manuel du guerrier de la lumière & Indisponible \\
\hline Onze minutes & Indisponible \\
\hline Sur le bord de la rivière Piedra, je me suis assise et j'ai pleuré & Indisponible \\
\hline Véronika décide de mourir & Indisponible \\
\hline
\end{tabular}

$* *$ Lecture du formulaire $\mathrm{n}^{\circ} 2$ :

Aux questions 1 et 2, les réponses sont respectivement : "Oui, bien évidemment. L'alchimiste et bien d'autres." et "Bien sûr! ". Quant à la question 3, la réponse est également L'alchimiste. La question 5, elle, reçoit une réponse bien mitigée. L'écriture fictionnelle et les thèmes abordés constituent, selon cette lectrice chevronnée (qui a déjà traduit du russe en français), un ensemble d'elmots concourant à un tel succès. Nous avons fait le constat que ladite librairie, symbolique dans cette zone, se trouvait dans une phase de transition. Ce qui justifie, selon sa libraire, le non ravitaillement de certains stands, y compris ceux abritant les ouvres de Paulo Coelho.

$$
\text { CARREFOUR SILOE - Cocody Saint Jean }
$$




\begin{tabular}{|l|r|}
\hline \multicolumn{1}{|c|}{ TITRES DISPONIBLES } & QTE EN STAND \\
\hline Adultère & 8 \\
\hline Aleph & Indisponible \\
\hline Amour & Indisponible \\
\hline Brida & Indisponible \\
\hline Comme le fleuve qui coule & Indisponible \\
\hline Hippie & Indisponible \\
\hline L'alchimiste & Indisponible \\
\hline La cinquième montagne & Indisponible \\
\hline La solitude du vainqueur & Indisponible \\
\hline La sorcière de Portobello & Indisponible \\
\hline Le démon et mademoiselle Prym & Indisponible \\
\hline Le manuscrit retrouvé & Indisponible \\
\hline Le pèlerin de Compostelle & Indisponible \\
\hline Le zahir & Indisponible \\
\hline L'espionne & Indisponible \\
\hline Maktub & Indisponible \\
\hline Manuel du guerrier de la lumière & Indisponible \\
\hline Onze minutes & Indisponible \\
\hline Sur le bord de la rivière Piedra, je me suis assise et j'ai pleuré & Indisponible \\
\hline Véronika décide de mourir & Indisponible \\
\hline
\end{tabular}

**Lecture du formulaire $\mathrm{n}^{\circ} 3$ :

Les réponses fournies pour les questions 1 et 2 sont: "Oui, L'alchimiste" et "Pas vraiment". A la différence de la librairie FNAC Cap Nord, la réponse 3 stipule que bien que le succès se soit étendu à l'ensemble de ses œuvres, "L'adultère" et "L'alchimiste" restent en tête de liste. La vision générale que cette libraire nous livre de l'œuvre de Paulo Coelho (réponse 5), c'est celle d'une écriture étrange et floue où, estime-t-elle, l'auteur ne va pas au bout de ses idées. Au-delà de leur aspect obscur, les œuvres de Paulo Coelho ont imposé, à notre lectrice, un certain nombre de questionnements qui, nous dit-elle, l'ont laissée sur sa faim.

Librairie de France - Plateau

\begin{tabular}{|l|r|}
\hline \multicolumn{1}{|c|}{ TITRES DISPONIBLES } & QTE EN STAND \\
\hline Adultère & Indisponible \\
\hline Aleph & Indisponible \\
\hline Amour & Indisponible \\
\hline Brida & Indisponible \\
\hline Comme le fleuve qui coule & Indisponible \\
\hline Hippie & Indisponible \\
\hline L'alchimiste & Indisponible \\
\hline La cinquième montagne & Indisponible \\
\hline La solitude du vainqueur & Indisponible \\
\hline La sorcière de Portobello & Indisponible \\
\hline Le démon et mademoiselle Prym & Indisponible \\
\hline Le manuscrit retrouvé & Indisponible \\
\hline Le pèlerin de Compostelle & Indisponible \\
\hline Le zahir & Indisponible \\
\hline L'espionne & Indisponible \\
\hline Maktub & Indisponible \\
\hline Manuel du guerrier de la lumière & Indisponible \\
\hline Onze minutes & Indisponible \\
\hline Sur le bord de la rivière Piedra, je me suis assise et j'ai pleuré & Indisponible \\
\hline Véronika décide de mourir &
\end{tabular}


**Lecture du formulairen ${ }^{\circ} 4$ :

Aucune réponse n'a été fournie aux questions 1,2, 3 et 5 du fait du non-retour du libraire en charge de cette section de la Librairie. Néanmoins, ce que nous avions pu observer, en prélude de l'envoi du questionnaire, c'était la présence, dans les stands, d'un seul exemplaire de "Hippie".

\section{ELMOTS ET RÉCEPTION: QUELQUES RÉFLEXIONS}

La méconnaissance du métier de traducteur et sa non-valorisation nous plonge dans une ignorance de l'autre toutefois terrible. Cette impéritie qui n'est pas toujours le fait du lecteur, en fait un ingrat malgré lui de l'apport de l'autre. A la question: Avez-vous des auteurs traduits du portugais en français? Si la négative peut sembler frappante, c'est simplement parce que l'autre, le lusophone Paulo Coelho, bien que reconnu par ses lecteurs comme brésilien, n'est surtout pas lu pour sa "brésilinité".

Aux yeux de bon nombre de ses lecteurs, c'est tout simplement un écrivain au même titre que Molière, Dan Brown, Marcel Proust, Jean-Marie Adiaffi, Sédar Senghor, Wole Soyinka, Chinua Achebe ou Ernest Hemingway. Si chez le grand public, cette confusion, certes regrettable, peut être comprise, il y a matière à discuter quand elle s'étend aux spécialistes du livre et aux critiques littéraires. En ne prêtant pas l'attention qu'il faut à ces détails pourtant très visibles et imposants, c'est faire le choix de passer sous silence la présence de l'autre. L'autre chez qui l'on veut et peut s'abreuver. L'autre qui nous visite et nous rénove. L'autre dont la poignée de main reste sans interlocuteur.

La lecture de Paulo Coelho en Côte d'Ivoire est singulièrement suscitée par la recherche d'œuvre d'aide personnelle. En effet, dans cette société en proie aux crises et aux questions existentielles, les ouvrages de Paulo Coelho sont sources d'inspiration pour des solutions individualisées. Ces retrouvailles, par la médiation des lettres entre l'auteur brésilien et le lecteur ivoirien, seraient une illustration d'un autre aspect du dialogue Sud-Sud. Selon le Professeur Boa Thiémélé Ramsès5, qui a accepté de se soumettre à nos questions,les ouvrages de Paulo Coelho sont "de très bons livres qui aident à supporter la vie. Ils ont une vision positive de l'existence. Niveau de langage simple, accessible à tous, avec de courtes phrases. Bien sûr, ce lecteur ivoirien se réfère aux livres lus dans leur version française. Bien que médié par l'un des actuels "Nords", nous voici invités dans un dialogue Sud-Sud. La lecture de ces œuvres en Côte d'Ivoire en dit long sur les possibilités de coopérations que l'un ou l'autre des deux peuples peut enseigner.

Il n'y a nul doute que la présence-absente de "L'alchimiste", telle que démontrée par les tableaux de chacune des librairies, suscite des interrogations. La portée spirituelle que certains lecteurs considèrent, à tort6, comme "le côté obscur" de Paulo Coelhoest, n'en doutons point, l'un des facteurs clés du succès de ses ouvrages. C'est à croire que l'ipséité de l'être d'aujourd'hui, du sujet contemporain, dépend d'un repère, mieux, d'un commandement plus extérieur qu'intérieur. Cette crise, à son tour bien accrochée à la vertèbre de cet individu moderne, lui rongeant l'âme à petite bouchées, résulte d'une double conscience (DUBOIS, 2007) dilacérée. Il s'agit ici d'un être partagé entre l'hyper-connexion (du fait des nouvelles technologies) et l'hypo-connexion (par ce que haché par de violents vents d'infox), partagé entre une ségrégation soft et une appartenance volatile, assoiffé de modernité et très nostalgique d'un passé qui expire, passionné par l'opulence pressante et torturé par la patience que requiert la lecture d'un paragraphe. En d'autres termes, l'inconscience démontrée par le sujet de cette "double conscience" (certes distincte du contexte et de l'idée que W.E.B Dubois s'en faisait) se justifie largement par une attitude d'insouciance presque mécanique, fruit d'une délégation de pouvoirs. Cet "Autre" plénipotentiaire, au sens large du terme, à qui le sujet confie tout (y compris sa vie) peut ainsi le conduire vers la destinée de son choix. Il se robotise et perd toute capacité de projection de soi et de (re)structuration de la société. Soit,

C'est à partir de cette présence à moi d'autrui-sujet, dans et par mon objectité assumée, que nous pouvons comprendre l'objectivation d'autrui comme second moment de mon rapport à l'autre. En effet la présence d'autrui par-delà ma limite non révélée peut servir de motivation pour mon ressaisissement de moi-même en tant que libre ipséité. Dans la mesure où je me nie comme autrui et où autrui se manifeste d'abord, il ne peut 
se manifester que comme autrui, c'est-à-dire comme sujet par-delà ma limite, c'est-à-dire comme ce qui me limite. Rien en effet ne peut me limiter sinon autrui. Il apparaît donc comme ce qui, dans sa pleine liberté et dans sa libre projection vers ses possibles, me met hors de jeu et me dépouille de ma transcendance, en refusant de " faire avec " (au sens de l'allemand : mit-machen). Ainsi dois-je saisir d'abord et uniquement celle des deux négations dont je ne suis pas le responsable, celle qui ne vient pas à moi par moi. Mais dans la saisie même de cette négation surgit la conscience (de) moi comme moi-même, c'est-à-dire que je puis prendre une conscience explicite (de) moi en tant que je suis aussi responsable d'une négation d'autrui qui est ma propre possibilité. C'est l'explicitation de la seconde négation, celle qui va de moi à autrui. A vrai dire, elle était déjà là, mais masquée par l'autre, puisqu'elle se perdait pour faire apparaître l'autre. Mais précisément l'autre est motif pour que la nouvelle négation paraisse : car s'il y a un autrui qui me met hors-jeu en posant ma transcendance comme purement contemplée, c'est que je m'arrache à autrui en assumant ma limite. Et la conscience (de) cet arrachement ou conscience (d'être) le même par rapport à l'autre est conscience (de) ma libre spontanéité. Par cet arrachement même qui met l'autre en possession de ma limite, je jette déjà l'autre hors de jeu. (SARTRE, 1943, pp. 326-327)

C'est dire que l'objectif premier de l'horizon d'expectation, la Côte d'Ivoire donc dans le cadre de notre étude, c'est avant tout, à travers l'œuvre de Paulo Coelho, d'engager la course vers la rencontre du moi avec lui-même, de la reconquête de son pouvoir anthropocentré qui permettra, par la suite, d'établir avec autrui un nouveau contrat social7. C'est en cela que la littérature peut s'avérer transcendantale, transversale et transnationale.

De ce qui précède, nous faisons le constat que le lecteur ivoirien est passé d'une phase de non besoin (consommation) à celle d'une production déficitaire(voir tableaux ci-dessus). Autrement dit, le processus d'internationalisation de l'œuvre de Paulo Coelho semble s'être appuyé, mieux, s'être investi dans la production du besoin de consommation au niveau national. Et dans ce processus de fixation et de promotion, par-delà les librairies citées, les clubs privés axés sur le développement personnel, le coaching, le leadership et l'art oratoire, ont été et continuent d'être d'un concours indéniable.

\section{CONCLUSION}

Les ouvrages de Paulo Coelho traduits en français sont reçus en Côte d'Ivoire où ils sont précédés par l'étiquette de best-sellers. Ils sont achetés et lus par un public restreint qui les découvrent dans les rayons de librairies ou au cours des conversations entre amis. La médiation des circuits d'institutions françaises nous met face à deux réalités presque liées: a) Quelle est l'identité et/ou la place du livre étranger en Côte d'Ivoire? Tous les grands classiques sont lus et étudiés dans différentes sphères sans être jamais perçus ou considérés comme des traductionset ; b) le manque d'une attention particulière des maisons d'édition traditionnelles pour le produit "ouvrage-traduit". Outre les syllabaires et les Écritures qui sont régulièrement traduites dans plusieurs de nos langues locales (nationales), nous n'avons connaissance d'aucun classique littéraire écrit dans une quelconque langue (européenne ou africaine) qui ait fait l'objet d'une traduction conduite en Côte d'Ivoire. Ce manque d'intérêt s'explique par la non-distinction claire entre livres traduits (en français et importés) et livres produits directement sur place (en français par des auteurs ivoiriens ou africains francophones). C'est dire que nos éditeurs très conservateurs et traditionnels n'ont pas encore perçus les innombrables opportunités d'affaires (dans les domaines de la politique, de la spiritualité, de la culture et de l'économie) que le secteur de la traduction littéraire pourrait engendrer. Ce constat amer fait le portrait d'une insuffisance énorme présentée par la Côte d'Ivoires pour ce qui a trait à l'établissement de "relations littéraire et idéelles directes" avec d'autres cultures. Même si l'intérêt pour les livres de Paulo Coelho va grandissant, il ne contribue jusqu'ici pas à jeter une nouvelle lumière sur le secteur de la traduction. Ce qui donne à sa réception un caractère d'incomplétude. 


\section{RÉFÉRENCES BIBLIOGRAPHIQUES}

ACHEBE, C. Tout s'effondre. Traduite par Michel Ligny. Paris: Editions Présence Africaine, 2000.

ADIAFFI, J-M. La carte d'identité. Abidjan: ceda, 1980.

CASANOVA, P. The World Republic of Letters. Translated by DeBevoise M. B. Cambridge Massachusetts Harvard University Press, 2004.

CASANOVA, P. La langue mondiale. Traduction et domination. Paris: Seuil (Collection Liber), 2015.

COELHO, P. Comme le fleuve qui coule. trad. de Françoise Marchand Sauvagnargues. Paris : Flammarion, 2006.

DU BOIS, W.E.B. Of Our Spiritual Strivings. In: The Souls of Black Folk. Brent Hayes Edwards (Ed.). New York: Oxford University Press, 2007, pp. 714.

FONSECA, V. P. da S., \& VIEIRA, K. M. (2011). A biografia como acontecimento jornalístico. Libero, 14, 99-108.

MORAIS, F. $O$ Mago, Planeta, 2013. Disponible sur : https://www1.folha.uol.com.br/fsp/ilustrad/fq3105200809.htm

NAUMANN, M. and Heath, P. Literary Production and Reception. New Literary History, Vol. 8, no. 1, 1976, pp. 107-126.

ROMANCINI, R. Paulo Coelho, um autor singular: da "cultura das bordas" ao "centro". INTERCOM - Sociedade Brasileira de Estudos Interdisciplinares da Comunicação XXV Congresso Brasileiro de Ciências da Comunicação - Salvador/BA - 1 a 5 Set 2002.

SARTRES, Jean-Paul. L'être et le néant. Essai d'ontologie phénoménologique. Paris: Gallimard, 1943.

TEZZA, C. Literatura $\quad$ C biografia. Disponible à: www.cristovaotezza.com.br/textos/palestras/Literatura e biografia - Cristovão Tezza.pdf Consulté le $14 / 09 / 2019$.

THOMPSON, P. Martyn. "Reception theory and the interpretation of historical meaning." History and Theory. Vol. 32, n. 3 (Oct., 1993), pp. 248-272.

\section{NOTAS}

1 " vista da perspectiva técnica, a literatura é o exercício de um plágio formal de gêneros já solidamente constituídos na vida real da linguagem. ", p. 10.

2 Morais, Fernando: $O$ Mago, Planeta, 2013. Disponible sur: https://www1.folha.uol.com.br/fsp/ilustrad/fq3105200809.htm

3 Sodré (2009) cité par Fonseca \& Vieira (2011).

4 Nous remercions particulièrement Madame Boni Jacqueline, propriétaire de ladite librairie, qui, dans une spontanéité presqu'inusité, a toute de suite accepté de se prêter à notre exercice, nous fournissant toutes les informations sollicitées.

5 Entretien non publié.

6 Position que Romancini (2002) défend après une étude empirique qu'il a lui-même conduite auprès des lecteurs d'une bibliothèque relative au succès éditorial de Paulo Coelho. De cette étude, il conclut que le côté ésotérique n'est certainement pas ce qui attire les lecteurs, en tout cas, pas dans leur majorité.

7 Marx nous explique que la production et la consommation sont deux phénomènes liés par une relation dialectique.

8 Production produces consumption insofar as it creates (a) the material, the object to be consumed; (b) by way of the object, which is always a definite one, the manner of consumption; (c) the need for consumption, 
the urge to it, the "ability to consume," and thus a subject for the object. Marx gives as an example: "The object of art, as well as any other product, creates an artistic public, appreciative of beauty" (p. 25). But conversely, consumption also produces production, and does so inasmuch as it creates (a) the real product, for unlike a natural object, the object created by man only becomes a product when it has proved itself to be such in consumption: "the result of production is a product, not as the material embodiment of activity but only as an object for the active subject" [...]; consumption creates (b) the need for new production, "the ideal, inward, impelling cause which constitutes the prerequisite of production" (ibid.), and thus "the disposition of the producer" (p. 26): "It is clear that while production furnishes the material object of consumption, consumption provides the ideal object of production, as its image, its want, its impulse and its purpose" (p. 25). (Marx, in Naumann, 1976, p. 108).

9 Nous nous référons aux politiques nationales de promotion du livre étranger. Il s'agit ici d'une politique bidirectionnelle. La Côte d'Ivoire qui traduit l'autre chez qui elle voit une source intarissable de richesses. La littérature ivoirienne traduite à et pour l'extérieur. Elle se verrait ainsi engager dans une géostratégie littéraire qui, vu ses ambitions sous-régionales et continentales, reste inévitable à la fois sur le plan littéraire que numéraire. Par exemple, pour lire "Tout s'effondre" de Chinua Achebe, le lecteur ivoirien doit passer par la traduction française de l'œuvre. Donc par une médiation (traduction), elle-aussi, médiée (maison d'édition). 